\title{
Cancer cells can be tricked into self-destructing
}

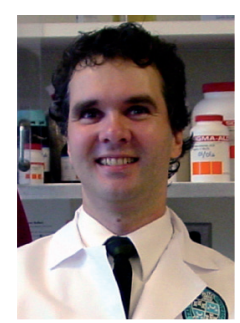

\author{
"Of the several routes one could take to \\ achieve cancer cell death, perhaps one
}

of the most poetic methods is to trick cancer

cells into performing the task themselves

by committing suicide."

\section{W T Godbey}

Laboratory for Gene Therapy \& Cellular Engineering, Department of Chemical \& Biomolecular Engineering,

Tulane University, 6823 St Charles Ave, 300 Lindy Boggs Center, New Orleans, LA 70178, USA

= Tel.: +1 5048655872 = Fax: +1 5048656744 = godbey@tulane.edu

To kill cancer cells is the ultimate objective of oncology researchers, but deciding exactly how to go about the task presents a wide array of logical approaches. Of the several routes one could take to achieve cancer cell death, perhaps one of the most poetic methods is to trick cancer cells into performing the task themselves by committing suicide. The differences between destruction via exogenously delivered cytotoxic agents, via agents that induce a secondary response within the host and via agents produced by the cells themselves can be nuanced, but the latter method holds promise for cancer cell death with minimal side effects. It is worth briefly discussing the different logical approaches to fully appreciate how cancer treatments have become more subtle as technology has progressed.

\section{"A word of caution for those considering expression targeting for anticancer gene therapy: it is not enough to run a microarray and select a gene that is highly expressed in a given tumor but not expressed in normal tissue."}

Common anticancer drugs, such as doxorubicin and mitomycin, which are anthracyclines, act directly upon dividing cells by halting replication. These drugs bind strands of DNA together to prevent replication (and transcription) and also interfere with topoisomerase II, a DNA replication enzyme. Cisplatin, which uses the heavy metal platinum, acts in a similar fashion by forming links with DNA to prevent replication. The key to these treatments is that they act upon dividing cells, and cancer cells are typically more mitotically active than untransformed cells. While these drugs have been used successfully to kill cancer cells, the cell death results from the cytotoxic drug that was delivered. The approach is direct and not subtle - there is no trickery at all. A more elegant method of obtaining cancer cell destruction is immunotherapy.

Immunotherapy includes approaches used to initiate immune attack. It is a means of harnessing the host's own immune system to attack cancer cells, and involves a form of communication, even negotiation, with the host's body; this can indeed be considered an artful approach. Such methods may utilize antibodies or adjuvants to stimulate immune attack, either directly against a certain cancer cell type or indirectly via a generalized activation of the immune system. An example of this approach is the drug trastuzumab, which specifically recognizes and binds to the HER2 protein, which is expressed on some types of breast and stomach cancers. The binding of the drug to cancer cells inhibits their growth and proliferation by preventing EGF binding, and the drug also triggers immune system attack upon the cancerous cells after binding. Another example is the drug bevacizumab, which is a monoclonal antibody against a specific type of VEGF (VEGF-A) and causes the inactivation and destruction of VEGF-A molecules [1]. VEGF-A plays a role in angiogenesis and destruction of the molecule will inhibit the development of tumors by inhibiting the creation of a new blood supply for the tissue. Similar to the examples previously given for cytotoxic agents, these drugs have been somewhat effective and, while they come closer to achieving the premise of this article in that they cause the body to take action against its own cancers, they fall short of having the cancer cells kill themselves. One class of immunostimulatory molecules, the interferons, have recently been shown to have an indirect effect upon cancer cell self-annihilation and thus offer a potentially finer approach to cancer treatment.

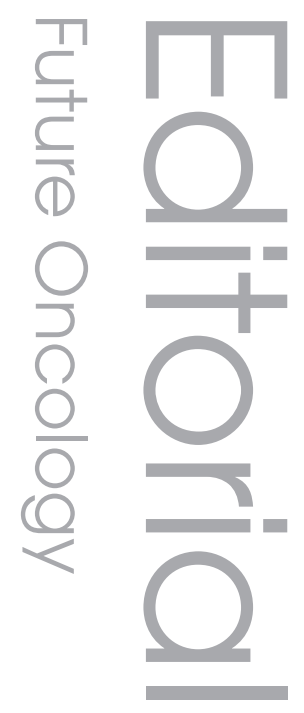


In the human body, interferons are cytokines that are made by lymphocytes to combat pathogens such as viruses or bacteria. They serve to activate cells of the immune system, such as macrophages and natural killer cells, and they increase antigen presentation so that foreign bodies (and sometimes tumor cells) can be more easily recognized. Interferons have also been used as a medical treatment for cancer. The logic behind their use originally focused upon the interaction of the drug with the immune system, but recent findings indicate that the drugs can affect cancer cells directly. Interferons, while inhibiting cellular proliferation and activating the immune system, also upregulate the expression of certain genes that, in turn, activate proapoptotic proteins [2]. This recent finding of gene activation is an indirect form of having cancer cells self-destruct. However, the upregulation is one step away from initiating apoptosis. A more direct method of having cancer cells self-destruct would be to deliver an apoptotic intermediate.

Cells undergoing apoptosis activate a cascade of suicide genes to effect their own selfdestruction while packaging their contents so that neighboring cells remain unaffected. Because apoptosis is an intracellular event with vesicularized degradation products, it is a promising choice for instituting cancer cell death with no bystander effect. The apoptosis cascade is primarily made up of proteins known as caspases. The higher-numbered caspases (caspase 8 [FLICE], caspase 9 [Apaf-3] and caspase 10 [Mch4]) are initiators, while the lower-numbered caspases (caspase 1 [ICE], caspase $2[\mathrm{ICH}-1]$ and caspase 3 [CPP32, Yama and Apopain]) are effectors.

At this point, one might conclude that delivering an apoptotic intermediate to cancer cells would be an effective way of inducing cancer cell death. However, problems exist in having the protein cross the plasma membrane into the cell, and in having the protein become activated for apoptosis. Instead, one could deliver proteins (e.g., the Fas ligand or TNF- $\alpha$ ) that initiate apoptosis by binding to extracellular receptors, but normal cells may also have the same receptors. In addition, many cancer cells have rendered apoptosis-resistant themselves by ceasing the production of such receptors. An effective way around these quandaries is to deliver genes that code for apoptotic intermediates. Genes for both initiator and effector caspases have been used in gene delivery experiments in an attempt to induce apoptosis [3-5]. An additional feature has been added to some caspase transgenes to render their protein products exogenously inducible, thus creating 'artificial death switches' [6]. The method is effective at bringing about self-destruction in transfected cells at a time determined by the caregiver. However, this is not the end of the story.

Having cells kill themselves is not enough for an effective cancer therapy. Targeting is, as it always has been, an issue. One could easily deliver benzene or rattlesnake venom to an area, but the amount of ancillary damage to healthy tissue would render such therapy no better than amputation, or worse. The cytotoxic drugs doxorubicin and mitomycin, discussed in the beginning of this article, have great potency in rapidly dividing cells, but this type of targeting is not restrictive enough to prevent the side effects associated with these chemotherapeutics since cells of the bone marrow, gut epithelium and hair follicles also divide actively. For gene therapy, there exist two classes of targeting that are available: ligand targeting and expression targeting. Ligand targeting involves the attachment of a ligand or antibody to the gene delivery vector. For example, antibodies against the transferrin receptor have been conjugated to polyethylene glycol and incorporated into liposomes to target transferrin receptor-expressing cancer cells [7]. Similarly, because expression of folate receptors can be a marker for certain types of cancer, folate has been conjugated to genedelivery agents to target cells that express folate receptors (reviewed in [8]). A caveat of any form of ligand targeting is that once the ligand (or receptor) has been attached to the gene therapy complex, certain physical parameters important to gene delivery are altered. To get around this alteration, the technique of expression targeting has been developed.

\section{"...the very gene that some cancer cells use to avoid apoptotic cell death is the one that is used to drive the expression of apoptotic proteins, inducing immortal cancer cells to kill themselves."}

Expression targeting involves the modification of the transgene so that only the cells of interest will express it after delivery. Such targeting is achieved through the use of regulatory units for DNA transcription such as promoters or enhancers. As an example, consider the gene for prostate-specific antigen $(p s a)$. The gene is regulated by the $p s a$ promoter. A prostate cancer 
cell expressing the $p s a$ gene will have transcriptional proteins that recognize the psa promoter. The trick comes when one uses the $p s a$ promoter to drive the expression of a delivered transgene. If one were to construct a gene that used the psa promoter to direct the expression of an exon coding for the green fluorescent protein, then one might expect green fluorescent prostate cancer cells following gene delivery. However, if the same transgene were delivered to, say, retinal cells, the gene should not be expressed because retinal cells lack the transcriptional proteins that recognize the psa promoter. A word of caution for those considering expression targeting for anticancer gene therapy: it is not enough to run a microarray and select a gene that is highly expressed in a given tumor but not expressed in normal tissue. Our laboratory has found that other factors must be considered, such as the mechanism behind upregulated gene expression [Dobeк, Zhang, Balazs and Godbey, Manuscript Submitted].

We have found that the promoter for cyclooxygenase type 2 (cox-2) works well for expression targeting in carcinoma cells, including bladder tumors [9,10]. Cox-2 is expressed by cells as part of the inflammatory pathway, but it is highly overexpressed by carcinoma cells

\section{Bibliography}

1. Los M, Roodhart JM, Voest EE: Target practice: lessons from Phase III trials with bevacizumab and vatalanib in the treatment of advanced colorectal cancer. Oncologist 12, 443-450 (2007).

2. Stawowczyk M, Van Scoy S, Kumar KP, Reich NC: The interferon stimulated gene 54 promotes apoptosis. J. Biol. Chem. DOI: 10.1074/jbc.M110.207068 (2010) (Epub ahead of print).

3. Shariat SF, Desai S, Song W et al.: Adenovirus-mediated transfer of inducible caspases: a novel 'death switch' gene therapeutic approach to prostate cancer. Cancer Res. 61, 2562-2571 (2001). as neovascularity is recruited to a developing tumor. It is also thought to play a part in apoptosis resistance and multidrug resistance in many cancers [11]. The poetry that was alluded to in the first paragraph is illustrated by this method: the very gene that some cancer cells use to avoid apoptotic cell death is the one that is used to drive the expression of apoptotic proteins, inducing immortal cancer cells to kill themselves. While expression-targeted gene therapy is still a long way from entering the cancer clinic, it is nonetheless a promising idea that is being intensely investigated. The trickery that is involved in obtaining cancer cell death yields a straightforward and elegant approach, with cancer cells performing the difficult work themselves.

\section{Financial \& competing interests disclosure \\ This work was funded by the National Science Foundation CAREER award (CBET-0846395). The author has no other relevant affiliations or financial involvement with any organization or entity with a financial interest in or financial conflict with the sub- ject matter or materials discussed in the manuscript apart from those disclosed. \\ No writing assistance was utilized in the production of this manuscript.}

4. Shinoura N, Sakurai S, Asai A, Kirino T, Hamada H: Caspase-9 transduction overrides the resistance mechanism against $\mathrm{p} 53$ mediated apoptosis in U-87MG glioma cells. Neurosurgery 49, 177-186 (2001).

5. Shinoura N, Sakurai S, Asai A, Kirino T, Hamada H: Transduction of Apaf-1 or caspase-9 induces apoptosis in A-172 cells that are resistant to $\mathrm{p} 53$-mediated apoptosis. Biochem. Biophys. Res. Commun. 272, 667-673 (2000).

6. Fan L, Freeman KW, Khan T, Pham E, Spencer DM: Improved artificial death switches based on caspases and FADD. Hum. Gene Ther. 10, 2273-2285 (1999).

7. Yu W, Pirollo KF, Rait A et al:: A sterically stabilized immunolipoplex for systemic administration of a therapeutic gene. Gene Ther. 11, 1434-1140 (2004).
8. Zhao X, Li H, Lee RJ: Targeted drug delivery via folate receptors. Expert Opin. Drug Deliv. 5, 309-319 (2008).

9. Zhang X, Atala A, Godbey WT: Expression-targeted gene therapy for the treatment of transitional cell carcinoma. Cancer Gene Ther. 15, 543-552 (2008).

10. Zhang X, Godbey WT: Preclinical evaluation of a gene therapy treatment for transitional cell carcinoma. Cancer Gene Ther. 18, 34-41 (2011).

11. Liu B, Qu L, Tao H: Cyclo-oxygenase 2 up-regulates the effect of multidrug resistance. Cell Biol. Int. 34, 21-25 (2009). 